\title{
Fermion Condensation Quantum Phase Transition versus Conventional Quantum Phase Transitions
}

\author{
V.R. Shaginyan ${ }^{a, b *}$, J.G. $\operatorname{Han}^{b}$, J. Lee ${ }^{b}$ \\ ${ }^{a}$ Petersburg Nuclear Physics Institute, Gatchina, 188300, Russia \\ ${ }^{b}$ CAPST, SungKyunKwan University 300, Chun-Chun-Dong Jangan-gu Suwon 44-746, Korea
}

\begin{abstract}
The main features of fermion condensation quantum phase transition (FCQPT), which are distinctive in several aspects from that of conventional quantum phase transition (CQPT), are considered. We show that in contrast to CQPT, whose physics in quantum critical region is dominated by thermal and quantum fluctuations and characterized by the absence of quasiparticles, the physics of a Fermi system near FCQPT or undergone FCQPT is controlled by the system of quasiparticles resembling the Landau quasiparticles. Contrary to the Landau quasiparticles, the effective mass of these quasiparticles strongly depends on the temperature, magnetic fields, density, etc. This system of quasiparticles having general properties determines the universal behavior of the Fermi system in question. As a result, the universal behavior persists up to relatively high temperatures comparatively to the case when such a behavior is determined by CQPT. We analyze striking recent measurements of specific heat, charge and heat transport used to study the nature of magnetic fieldinduced QCP in heavy-fermion metal $\mathrm{CeCoIn}_{5}$ and show that the observed facts are in good agreement with our scenario based on FCQPT and certainly seem to rule out the critical fluctuations related with CQPT. Our general consideration suggests that FCQPT and the emergence of novel quasiparticles near and behind FCQPT and resembling the Landau quasiparticles are distinctive features intrinsic to strongly correlated substances.
\end{abstract}

PACS: 71.10.Hf; 71.27.+a; 74.72.-h

Keywords: Quantum phase transitions; Heavy fermions; Transport properties

Typeset using REVTEX

*E-mail: vrshag@thd.pnpi.spb.ru 
It is generally accepted that the fundamental physics that gives rise to the high- $T_{c}$ superconductivity and non-Fermi liquid (NFL) behavior with a recovery of the Landau-Fermi liquid (LFL) behavior under the application of magnetic fields observed in heavy-fermion (HF) metals and high- $T_{c}$ compounds is controlled by quantum phase transitions. This has made quantum phase transitions a subject of intense current interest, see e.g. [1,2].

A quantum phase transition is driven by control parameters such as composition, density or magnetic fields and takes place at a quantum critical point (QCP) when temperature $T=0$. QCP separates an ordered phase generated by quantum phase transition from a disordered phase. It is expected that the universal behavior is only observable if the system in question is very near to QCP, for example, when the correlation length is much larger than microscopic length scales. Quantum phase transitions of this sort are quite common, and we shall label such quantum phase transitions as conventional quantum phase transitions (CQPT). In the case of CQPT, the physics is dominated by thermal and quantum fluctuations of the critical state, which is characterized by the absence of quasiparticles. It is believed that the absence of quasiparticle-like excitations is the main cause of the NFL behavior and other types of the critical behavior in the quantum critical region. Basing on the assumption of scaling related to the divergency of the correlation length, one can construct the critical contribution to the free energy and evaluate the corresponding properties such as critical exponents, the NFL behavior, etc. [1,2]. Moving along this way, one may expect difficulties. For example, having the only critical contribution, one has to describe at least two types of the behavior exhibited by different HF metals, see e.g. [3-5]. Note that HF metals are three-dimensional structures, see e.g. [3,6-8] and, thus, the type of behavior cannot be related to the dimension. The critical behavior observed in measurements on HF metals takes place up to rather high temperatures comparable with the effective Fermi temperature $T_{k}$. For example, the thermal expansion coefficient $\alpha(T)$ measured on $\mathrm{CeNi}_{2} \mathrm{Ge}_{2}$ shows a $1 / \sqrt{T}$ divergence over more than two decades in temperature from $6 \mathrm{~K}$ down to at least $50 \mathrm{mK}$ [3]. It is hardly possible to understand such a behavior basing on the assumption of scaling when the correlation length is to be much larger than microscopic length scales. Obviously, such a situation can only take place at $T \rightarrow 0$. At $T \sim T_{k}$, this macroscopically large correlation length must be destroyed by thermal fluctuations. The next problem is related to explanations of recovery of the LFL behavior under applied magnetic fields $B$ observed in HF metals. At $T \rightarrow 0$, the magnetic field dependence of the coefficient $A(B)$, the Sommerfeld coefficient $\gamma(B)$ and $\chi(B)$ in the resistivity $\rho=\rho_{0}+\Delta \rho, \Delta \rho=A(B) T^{2}$, specific heat, $C / T=\gamma(B)$, and magnetic susceptibility, $\chi(B)$, shows that $A(B) \sim \gamma(B)^{2}$ and $A(B) \sim \chi(B)^{2}$, so that the Kadowaki-Woods ratio, $K=A(B) / \gamma^{2}(B)$ [7], is conserved [8]. Such a universal behavior is hardly possible to explain within the picture assuming the absence of quasiparticles which takes place near QCP of the corresponding CQPT. As a consequence, for example, these facts are in variance to the spin-density-wave scenario [8] and the renormalization group treatment of quantum criticality [9]. Moreover, striking recent measurements of specific heat, charge and heat transport used to study the nature of magnetic field-induced QCP in heavy-fermion metal CeCoIn $5[10,11]$ certainly seem to disagree with descriptions based on CQPT.

In this Letter, we consider the main features of fermion condensation quantum phase transition (FCQPT), which is distinctive in several aspects from CQPT. We show that in contrast to CQPT, whose physics in the quantum critical region is dominated by thermal or quantum fluctuations and characterized by the absence of quasiparticles, the physics of a Fermi system near FCQPT or undergone FCQPT is controlled by the system of quasiparticles resembling the Landau quasiparticles. Since FCQPT is characterized by the superconducting order parameter $\kappa(\mathbf{p})$ [12], the critical region of fluctuations of $\kappa(\mathbf{p})$ is extremely narrow at $T \rightarrow 0$ and therefore it gives a negligible small critical contribution to the free energy. Contrary to the Landau quasiparticles, the effective mass of these 
quasiparticles strongly depends on the temperature, magnetic fields, density, etc. This system of quasiparticles having general properties determines the universal behavior of the Fermi system in question at finite temperatures and in magnetic fields being the main cause of the NFL behavior and other types of the critical behavior. As a result, the universal behavior persists up to relatively high temperatures and magnetic fields comparatively to the case when such a behavior were determined by CQPT. We analyze striking recent measurements of specific heat, charge and heat transport which where used to study the nature of magnetic field-induced QCP in heavy-fermion metal $\mathrm{CeCoIn}_{5}$ $[10,11]$ and show that the observed facts are in good agreement with our scenario based on FCQPT.

In the LFL theory, the existence of low-energy elementary excitations is the general property of a Fermi liquid and these excitations are represented by quasiparticles [13]. The quasiparticle distribution function $n(\mathbf{p}, T)$ is given by the equation

$$
\frac{\delta \Omega}{\delta n(\mathbf{p}, T)}=\varepsilon(\mathbf{p}, T)-\mu(T)-T \ln \frac{1-n(\mathbf{p}, T)}{n(\mathbf{p}, T)}=0 .
$$

The function $n(\mathbf{p}, T)$ depends on the momentum $\mathbf{p}$ and the temperature $T$. Here $\Omega=E-T S-\mu N$ is the thermodynamic potential, and $\mu$ is the chemical potential, while $\varepsilon(\mathbf{p}, T)$,

$$
\varepsilon(\mathbf{p}, T)=\frac{\delta E[n(p)]}{\delta n(\mathbf{p}, T)}
$$

is the quasiparticle energy. This energy is a functional of $n(\mathbf{p}, T)$ just like the total energy $E[n(p)]$, entropy $S[n(p)]$ and the other thermodynamic functions. The entropy $S[n(p)]$ is given by the familiar expression

$$
S[n(p)]=-2 \int[n(\mathbf{p}, T) \ln n(\mathbf{p}, T)+(1-n(\mathbf{p}, T)) \ln (1-n(\mathbf{p}, T))] \frac{d \mathbf{p}}{(2 \pi)^{3}},
$$

which results from purely combinatorial considerations. Eq. (1) is usually presented as the FermiDirac distribution

$$
n(\mathbf{p}, T)=\left\{1+\exp \left[\frac{(\varepsilon(\mathbf{p}, T)-\mu)}{T}\right]\right\}^{-1} .
$$

At $T \rightarrow 0$, one gets from Eqs. (1), (4) the standard solution $n_{F}(\mathbf{p}, T \rightarrow 0) \rightarrow \theta\left(p_{F}-p\right)$, with $\varepsilon\left(p \simeq p_{F}\right)-\mu=p_{F}\left(p-p_{F}\right) / M_{L}^{*}$, where $p_{F}$ is the Fermi momentum, $\theta\left(p_{F}-p\right)$ is the step function, and $M_{L}^{*}$ is the Landau effective mass [13]

$$
\frac{1}{M_{L}^{*}}=\left.\frac{1}{p} \frac{d \varepsilon(p, T \rightarrow 0)}{d p}\right|_{p=p_{F}} .
$$

It is implied that in the case of LFL $M_{L}^{*}$ is positive and finite at the Fermi momentum $p_{F}$. As a result, the $T$-dependent corrections to $M_{L}^{*}$, to the quasiparticle energy $\varepsilon(p)$, and to other quantities, start with $T^{2}$-terms being approximately temperature independent.

There exist special solutions of Eq. (1) associated with the so-called fermion condensation [14,15]. Being continuous and satisfying the inequality $0<n_{0}(\mathbf{p})<1$ within some region in $p$, such solutions $n_{0}(\mathbf{p})$ admit a finite limit for the logarithm in Eq. (1) at $T \rightarrow 0$ yielding $[14,15]$

$$
\varepsilon(\mathbf{p})-\mu=0, \quad \text { if } \quad 0<n_{0}(\mathbf{p})<1 ; p_{i} \leq p \leq p_{f} \text {, }
$$


where $\varepsilon(\mathbf{p})$ is given by Eq. (2). At $T=0$, Eq. (6) defines a new state of electron liquid with FC [14-16], which is characterized by a flat spectrum in the $\left(p_{f}-p_{i}\right)$ region, and which can strongly influence measurable quantities up to temperatures $T \ll T_{f}$. Here $T_{f}$ stands for the temperature where the fermion condensate effects disappear, $T_{f} / \varepsilon_{F} \sim\left(p_{f}-p_{i}\right) / p_{F}$, [15]. Here $\varepsilon_{F} \sim p_{F}^{2} / M_{L}^{*}$ is the Fermi energy. Note that at $T \ll T_{f}$ the occupation numbers of quasiparticles is approximately temperature independent, $n(\mathbf{p}, T) \simeq n_{0}(\mathbf{p})$, with $n_{0}(\mathbf{p})$ being given by Eq. (6). At $T=0$ in the state with FC, the order parameter coincides with the order parameter of superconducting state $\kappa(\mathbf{p})=\sqrt{\left(1-n_{0}(\mathbf{p})\right) n_{0}(\mathbf{p})}$ and has finite values in the $\left(p_{f}-p_{i}\right)$ region. While, the maximum value of the superconducting gap $\Delta_{1} \rightarrow 0$ in this region and the transition temperature $T_{c} \rightarrow 0$, provided that the pairing interaction tends to zero. Such a state can be considered as superconducting, with an infinitely small value of $\Delta_{1}$, so that the entropy $S(T=0)$ of this state is equal to zero $[12,15,17]$.

When $p_{f} \rightarrow p_{i} \rightarrow p_{F}$ the flat part vanishes, and Eq. (6) determines QCP at which the effective mass $M^{*}$ diverges due to density and spin fluctuations [15,18-23]. FCQPT manifests itself in the divergence of the quasiparticle effective mass $M^{*}$ as the density $x$ tends to the critical density $x_{F C}$, or the distance $r=\left(x-x_{F C}\right) \rightarrow 0$ being positive [21-23]

$$
M^{*} \propto \frac{1}{x-x_{F C}} \propto \frac{1}{r}
$$

As long as the effective mass $M^{*}$ is finite, the system exhibits the LFL behavior at low temperatures $T \sim T^{*}(x) \propto\left|x-x_{F C}\right|^{2}[23]$. The behavior of $M^{*}$ given by Eq. (7) is in good agreement with experimental facts obtained both in measurements on 2D Fermi systems such as electron gas and ${ }^{3} \mathrm{He}$ [24-26] and recent calculations [27-29]. We note that at $x \rightarrow x_{F C}$, the quasiparticle renormalization factor $z$ remains approximately constant and the divergence of the effective mass $M^{*}$ is not related to vanishing $z$, see e.g. $[21,28,30]$. Thus, Eq. (6) possesses non-trivial solutions at the critical quantum point $x=x_{F C}$ as soon as the kinetic energy $E_{k} \sim p_{F}^{2} / M^{*}$ becomes frustrated and the effective inter-electron interaction, or the Landau amplitude, being sufficiently large, start to determine the occupation numbers $n(\mathbf{p})$ which deliver the minimum value to the energy $E[n(p)]$. As a result, the occupation numbers $n(\mathbf{p})$ become variational parameters and Eq. (6) has non-trivial solutions $n_{0}(\mathbf{p})$, because the energy $E[n(p)]$ can be lowered by alteration of the occupation numbers. Thus, within the region $p_{i}<p<p_{f}$, the solution $n_{0}(\mathbf{p})$ deviates from the Fermi step function $n_{F}(\mathbf{p})$ in such a way that the energy $\varepsilon(\mathbf{p})$ stays constant, while outside this region $n_{0}(\mathbf{p})$ coincides with $n_{F}(\mathbf{p})[14,15]$. In response to this, the system becomes divided into two quasiparticle subsystems: the first subsystem in the $\left(p_{f}-p_{i}\right)$ range is characterized by quasiparticles with the effective mass $M_{F C}^{*} \rightarrow \infty$, while the second one is occupied by quasiparticles with finite mass $M_{L}^{*}$ and momenta $p<p_{i}$. It is seen from Eq. (7) that in the absence of FCQPT the effective mass could become negative at $r<0$. Thus, forming $\mathrm{FC}$ below the critical point $x_{F C}$, the system escapes the possibility to be in meaningless states with negative values of the effective mass. We note, that a formation of the flat part of the spectrum has been observed in [31,32] and obtained within exactly solvable models as well [33].

At $\Delta_{1} \rightarrow 0$, the critical temperature $T_{c} \rightarrow 0$. We see that the ordered phase with the order parameter $\kappa(\mathbf{p})$ and FC can exist only at $T=0$, and the state of electron liquid with FC disappears at $T>0[12,17]$. Therefore, FCQPT is not the endpoint of a line of finite-temperature phase transitions. This conclusion is in accordance with Eq. (1) which does not admit the existence of the flat part of spectrum at finite temperatures. FCQPT is driven by the divergency of the effective mass of quasiparticles. To put it differently, at QCP when $x \rightarrow x_{F C}$, the effective mass $M^{*}$ diverges due to both density and spin fluctuations, and in its turn, making the kinetic energy be frustrated, drives FCQPT with the superconducting order parameter $\kappa(\mathbf{p})$. Importantly, that the 
density and spin fluctuations in the question are not the critical fluctuations and the divergency of the effective mass is not a result of the critical fluctuations or vanishing the quasiparticle renormalization factor $z[19,21,23,28,30]$. We remark that in the case of the superconducting phase transition when $T_{c} / \varepsilon_{F} \ll 1$, the critical region of the critical fluctuations of the order parameter is so narrow that is almost irrelevant from the experimental point of view [34]. Thus, we can conclude that at finite temperatures $T>T_{c}$ the physics of Fermi system near FCQPT or behind the critical point of FCQPT is controlled by the system of quasiparticles resembling the Landau quasiparticles. Therefore, at $T_{c} \rightarrow 0$, the area of applications of the quasiparticle scenario extends practically to $T=0$ because the critical region becomes very narrow and eventually vanishes.

Assume that $T_{c} \rightarrow 0$ and $r<0$, then at finite temperatures $T \ll T_{f}$, the occupation numbers in the region $\left(p_{f}-p_{i}\right)$ are still determined by Eq. (6), and the quasiparticle system becomes divided into two quasiparticle subsystems: the first subsystem is occupied by normal quasiparticles with the finite effective mass $M_{L}^{*}$ independent of $T$ at momenta $p<p_{i}$, while the second subsystem in the $\left(p_{f}-p_{i}\right)$ range is characterized by the quasiparticles with the effective mass $M_{F C}^{*}(T)[12,15,17]$

$$
M_{F C}^{*} \simeq p_{F} \frac{p_{f}-p_{i}}{4 T}
$$

There is an energy scale $E_{0}$ separating the slow dispersing low energy part, related to the effective mass $M_{F C}^{*}$, from the faster dispersing relatively high energy part, defined by the effective mass $M_{L}^{*}$. It follows from Eq. (8) that $E_{0}$ is of the form [12]

$$
E_{0} \simeq 4 T \text {. }
$$

At $r<0$, the Fermi system can be viewed as a strongly correlated one demonstrating the NFL behavior even at low temperatures as it follows from Eqs. (8) and (9). At $T \rightarrow 0$, it behaves as a Fermi system moving towards a quantum critical point, for example, the effective mass diverges, $M_{F C}^{*} \propto 1 / T$, while the Grüneisen ratio, $\Gamma(\mathrm{T})=\alpha(T) / C(T)$, diverges algebraically, $\Gamma(\mathrm{T}) \propto 1 / \sqrt{T}$ $[4,5]$. Here, $\alpha(T)$ is the thermal expansion coefficient and $C(T)$ is the specific heat. In fact, at $T \rightarrow 0$, the system approaches the FC quantum critical line from above, and its behavior is controlled by the system of quasiparticles with the effective masses $M_{F C}^{*}$ and $M_{L}$. At $T \rightarrow 0$, the Fermi system in question is undergone a weakly first-order quantum phase transition because the entropy is not a continuous function at $T=0: \delta S=S(T>0)-S(T=0)$ is finite since $S(T=0)=0$, and at $0<T \ll T_{f}$, the entropy possesses the finite contribution coming from the occupations numbers $n_{0}(\mathbf{p})$, see Eqs. (3) and (6). On the other hand, according to the well known inequality, $\delta Q \leq T \delta S$, the heat $\delta Q$ of the transition is equal to zero, because $T=T_{c} \rightarrow 0$. In the same way, other thermodynamic functions such as the thermodynamic potential $\Omega$ and the free energy, $F=E-T S$, are continuous functions at $T \rightarrow 0$. Actually, FC cannot survive at $T \rightarrow 0$ because of the degeneracy of the FC spectrum, being absorbed by phase transitions removing the degeneracy. For example, FCQPT can be absorbed by the superconducting phase transition, see below. In that case, the critical temperature $T_{c}$ becomes finite, and at $T \rightarrow T_{c}$, we have the continues phase transition. It is pertinent to note that contrary to the considered case, a conventional quantum phase transition, at its quantum critical line, makes Grüneisen ratio $\Gamma(\mathrm{T})$ diverge at most logarithmically, $\Gamma(\mathrm{T}) \propto \pm \log T$, [35].

At $T \geq 0$ and $r=\left(x-x_{F C}\right)>0$, the system is on the disordered side and the effective mass given by Eq. (7) becomes finite. As a result, the kinetic energy comes into a play and makes the flat part vanish. Obviously, at $T=0$, Eq. (6) has only the trivial solution $\varepsilon\left(p=p_{F}\right)=\mu$, and the quasiparticle 
occupation numbers are given by the step function, $n_{F}(\mathbf{p})=\theta\left(p_{F}-p\right)$. At $\varepsilon_{F} \gg T>T^{*}(x)$ and $\left|x-x_{F C}\right| / x_{F C} \ll 1$, the effective mass $M^{*}$ depends on the temperature $[5,23]$

$$
M^{*}(T) \propto \frac{1}{\sqrt{T}}
$$

The state of system with $M^{*}$ strongly depending on $T$ and $r$ resembles the strongly correlated liquid. In contrast to the strongly correlated liquid, there is no energy scale $E_{0}$ given by Eq. (9). Such a system can be viewed as a highly correlated liquid and becomes the Landau Fermi liquid at $T \rightarrow 0$. We expect that Eq. (9) is valid up to temperatures $T \sim T_{k} \ll \varepsilon_{F}[5,23]$.

The LFL behavior is restored by the application of magnetic field $B>B_{c 0}$ and $T$-dependent corrections to the effective mass begin with $T^{2}$-terms. Here $B_{c 0}$ is a critical field which suppresses the magnetically ordered state and can be as big as 10-12 $\mathrm{T}$ [36] and even bigger. If the magnetically ordered state is absent then $B_{c 0}=0$, as it takes place in the case of $\mathrm{CeRu}_{2} \mathrm{Si}_{2}[5,37]$. At $r>0$ and $T^{*}(B)>T$, the effective mass $M^{*}(B)$ of the restored LFL depends on magnetic field $B[5,23]$

$$
M^{*}(B) \propto \frac{1}{\left(B-B_{c 0}\right)^{2 / 3}} .
$$

The function $T^{*}(B) \propto\left(B-B_{c 0}\right)^{4 / 3}$ determines the line on the $B-T$ phase diagram separating the region of the LFL behavior from the NFL behavior taking place at $T>T^{*}(B)[5,23]$. At $r<0$, a recovery of the LFL behavior under applied magnetic fields takes place at $T<T^{*}(B) \propto \sqrt{B-B_{c 0}}$, while the effective mass is given by [38]

$$
M^{*}(B) \propto \frac{1}{\sqrt{B-B_{c 0}}} .
$$

It follows from Eqs. (11) and (12) that the Kadowaki-Woods ratio $K=A(B) / \gamma^{2}(B)$ is conserved because $A(B) \propto\left(M^{*}(B)\right)^{2}$ and $\gamma(B) \propto M^{*}(B)$. Thus, the quasiparticle systems described by Eqs. $(8),(9),(10),(11)$, and (12) determine the universal behavior which is observed in measurements on HF metals [5].

To capture and summarize the salient features of magnetic field-tuned CQP observed recently in $\mathrm{CeCoIn}_{5}[10,11]$, we apply the above consideration based on FCQPT. A recent study of $\mathrm{CeCoIn}_{5}$ in magnetic fields $B>B_{c 0}$ have revealed that the coefficients $A(B)$ and $C(B)$, describing scattering in the LFL regime and determining the $T^{2}$ contributions to the resistivity $\rho$ and thermal resistivity $\kappa_{r}$ respectively, possess the same critical field dependence

$$
A(B) \propto C(B) \propto \frac{1}{\left(B-B_{c 0}\right)^{4 / 3}},
$$

with $B_{c 0}=5 \mathrm{~T}$, so that the ratio $A(B) / C(B)=c[11]$. Here $c$ is a field-independent constant characterizing electron-electron scattering in metals and having a typical value of 0.47 , see e.g. $[39,40]$. The observed critical exponent $4 / 3$ is in excellent agreement with that of given by Eq. (11) because $A(B) \propto C(B) \propto\left(M^{*}(B)\right)^{2}$. Such the parallel behavior of charge and heat transport with the scattering rate growing as $T^{2}$ shows that the delocalized fermionic excitations are the Landau quasiparticles carrying charge $e$. We note that these should be destroyed in the case of CQPT [1,2]. Nonetheless, let us assume for a moment that these survive. Since the heat and charge transport tend to strongly differ in the presence of the critical fluctuations of superconducting nature, the constancy of the ratio rules out the critical fluctuations [11]. On the other hand, one 
could expect that some kind of critical fluctuations could cause the observed behavior. For example, large scattering from antiferromagnetic fluctuations of finite momenta could degrade the heat and charge transport in a similar way [40]. In this case, in order to preserve the Kadowaki-Woods ratio these fluctuations are to properly influence the specific heat which characterizes the thermodynamic properties of the system and is not directly related to the transport one. On the other hand, there are no theoretical grounds for this. Therefore, the conservation of the Kadowaki-Woods ratio observed in recent measurements on $\mathrm{CeCoIn}_{5}$ [10] definitely seems to rule out these fluctuations. While both the constancy of Kadowaki-Woods ratio [10] and the constancy of the $A(B) / C(B)$ ratio [11] give strong evidence in favor of the quasiparticle picture. We remark that the above consideration of relationships between critical fluctuations and FCQPT is in agreement with these facts.

It is instructive to briefly analyze the behavior of the system when the magnetic field is changed through $B_{c 0}$. Broadly speaking, the magnetic field can be regarded as the tuning parameter (like e.g. pressure or density) which produces CQP, while $B_{c 0}$ can be regarded as the metamagnetic field at which FCQPT takes place. We remember that the behavior of system at FCQPT is not determined by critical fluctuations, therefore, the behavior is not disturbed by the proximity to first order phase transitions which could take place near the metamagnetic field. At $B<B_{c 0}$, we have to replace $\left(B-B_{c 0}\right)$ with $\left(B_{c 0}-B\right)$ in Eqs. (11) and (12) and in the formulas determining functions $T^{*}(B)$. Then it follows from Eqs. (11) and (12) that at $B=0$, the system in question exhibits the LFL behavior at temperatures $T<T^{*}\left(B_{c 0}\right)$. At elevated magnetic field and when $B \rightarrow B_{c 0}$ from below, the temperature $T^{*}(B)$ is depressed, $T^{*}(B) \rightarrow 0$, showing the reverse trend with respect to the case when $B>B_{c 0}$, so that the area of the LFL behavior vanishes. Therefore, it might be said that increasing magnetic field $B$ suppresses the LFL behavior enhancing the NFL behavior. As a result, in both cases $r<0$ and $r>0$, at the elevated magnetic field with $B<B_{c 0}$, the evolution of the effective mass $M^{*}$, specific heat $C$, resistivity $\rho$, etc, show the opposite trend with respect to the case when $B>B_{c 0}$. For example, at increasing magnetic field $B \rightarrow B_{c 0}$, the Sommerfeld coefficient $\gamma$ becomes divergent at $T \rightarrow 0$. We can conclude, that passing through the critical field $B=B_{c 0}$ leads to a sharp maximum in $M^{*}(B), \gamma(B), \rho(B), \chi(B)$, etc, see Eq. (11). While the Kadowaki-Woods ratio is conserved when the system exhibits the LFL behavior. Note that very near $B=B_{c 0}$ the temperature $T^{*}(B) \rightarrow 0$, therefore at finite temperatures, the sharp maximum is substituted by a relatively broad maximum which becomes sharper as $T \rightarrow 0$. A similar behavior to the described above was observed in measurements on $\mathrm{Sr}_{3} \mathrm{Ru}_{2} \mathrm{O}_{7}$ [41] and $\mathrm{CeIrIn}_{5}$ [42]. A detailed consideration of these items will be published elsewhere.

A few remarks related to the high- $T_{c}$ superconductivity are in order here. Let us discuss the situation in a finite external field as it is done in the case of continues phase transitions. Switch on the pairing interaction $\lambda V\left(\mathbf{p}_{1}, \mathbf{p}_{2}\right)$ generating the pairing field

$$
\Delta(\mathbf{p})=\lambda \int V\left(\mathbf{p}, \mathbf{p}_{1}\right) \kappa\left(\mathbf{p}_{1}\right) \frac{d \mathbf{p}_{1}}{(2 \pi)^{3}} .
$$

Here $\lambda$ is the coupling constant. The pairing field can be considered as an external field because the order parameter $\kappa(\mathbf{p})=\sqrt{\left(1-n_{0}(\mathbf{p})\right) n_{0}(\mathbf{p})}$ is determined by the strong Landau interaction rather then by the weak pairing interaction. We consider a weak coupling regime at which the occupation numbers of quasiparticles $n_{0}(\mathbf{p})$ are not disturbed by the pairing interaction and given by Eq. (6). The pairing field, being linearly coupled with the order parameter, see Eq. (14), removes the system from its critical point replacing FCQPT with the superconducting phase transition. In response to it, the FC plateau inclines with the slope being proportional to $\Delta_{1}$, while the effective mass becomes finite $M_{F C}^{*} \propto 1 / \Delta_{1}[12]$. In that case, it turns out that the maximum value of the superconducting 
gap $\Delta_{1}$ is linear with respect to small values of $\lambda[14,15,17]$ and can be as large as $\Delta_{1} \sim 0.1 \varepsilon_{F}$ and the transition temperature behaves as $T_{c} \propto x\left(x_{F C}-x\right)$ where $x$ stands for the doping level $[12,43]$. At finite temperatures $T \leq T_{c}$, the quasiparticle excitations are the Bogoliubov quasiparticles. Moving along this line, it is possible to explain the main features of the high- $T_{c}$ superconductivity including a recovery of the LFL behavior under applied magnetic fields as well, see e.g. $[12,14,15,43-45]$.

As the Landau theory of Fermi liquid, the theory of the high-temperature superconductivity based on FCQPT deals with the quasiparticles which are elementary excitations of low energies. This theory produces the general qualitative description of the superconducting state, normal one and the recovery of the LFL behavior under the application of magnetic field $[12,14,15,45]$. On the other hand, one can choose the phenomenological parameters and obtain the quantitative consideration of the superconductivity as it can be done in the framework of the Landau theory when describing a particular normal Fermi-liquid, say liquid ${ }^{3} \mathrm{He}$. Thus, any theory which is capable of describing FC and incorporates with the BCS theory will produce the qualitative picture of the superconducting state and the normal state which coincides with the picture based on FCQPT. Both of the pictures can agree at a numerical level provided the corresponding parameters are adjusted. For example, since the formation of flat band corresponding to FC is possible in the Hubbard model [32], one can, generally speaking, repeat the results of the theory based on FCQPT within the Hubbard model. It is appropriate mention here that the corresponding numerical description confined to the case of $T=0$ has been obtained within the Hubbard model $[46,47]$.

In conclusion, we have shown that in contrast to CQPT, whose physics is dominated by thermal and quantum fluctuations and characterized by the absence of quasiparticles, the physics of a Fermi system near FCQPT or undergone FCQPT is determined by quasiparticles resembling the Landau quasiparticles. Contrary to the Landau quasiparticles, the effective mass of these quasiparticles is strongly depends on the temperature, magnetic fields, density, etc. This system of quasiparticles has the general properties and determines the universal behavior of the Fermi system under consideration including the recovery of the LFL behavior under applied magnetic fields which preserves the Kadowaki-Woods ratio. This universal behavior persists up to relatively high temperatures comparatively to the case when such a behavior is determined by CQPT. We have analyzed the striking recent measurements of specific heat, charge and heat transport which where used to study the nature of magnetic field-induced QCP in heavy-fermion metal CeCoIn ${ }_{5}$ and shown that the observed facts are in good agreement with our scenario based on FCQPT and certainly seem to rule out the critical fluctuations related with CQPT. We have demonstrated that the Fermi system in question can be represented by the electronic systems of the high- $T_{c}$ superconductors, HF metals and by some two-dimensional Fermi systems. Finally, our general consideration suggests that FCQPT and the emergence of novel quasiparticles at QCP and behind QCP and resembling the Landau quasiparticles are qualities intrinsic to strongly correlated substances.

The authors are grateful for the financial support provided by the Korea Science and Engineering Foundation through the Center for Advanced Plasma Surface Technology at SungKyunKwan University. 


\section{REFERENCES}

[1] S. Sachdev, Quantum Phase transitions, Cambridge, Cambridge University Press, 1999.

[2] M. Vojta, Rep. Prog. Phys. 66 (2003) 2069.

[3] R. Küchler et al., Phys. Rev. Lett. 91 (2003) 066405.

[4] M.Ya. Amusia, A.Z. Msezane, V.R. Shaginyan, Phys. Lett. A 320 (2004) 459.

[5] V.R. Shaginyan, JETP Lett. 79 (2004) 344.

[6] H. Kadowaki, T. Fukura, K. Maezawa, Acta Phys. Pol. B 34 (2003) 375.

[7] K. Kadowaki, S.B. Woods, Solid State Commun. 58 (1986) 507.

[8] P. Gegenwart et al., Phys. Rev. Lett. 89 (2002) 056402.

[9] A.J. Millis, A.J. Schofield, G.G. Lonzarich, S.A. Grigera, Phys. Rev. Lett. 88 (2002) 217204.

[10] A. Bianchi et al., Phys. Rev. Lett. 91 (2003) 257001.

[11] J. Paglione et al., cond-mat/0405157.

[12] M.Ya. Amusia, V.R. Shaginyan, JETP Lett. 73 (2001) 232; S.A. Artamonov, V.R. Shaginyan, JETP 92 (2001) 287; M.Ya. Amusia, V.R. Shaginyan, Phys. Rev. B 63 (2001) 224507; V.R. Shaginyan, Physica B 312-313C (2002) 413.

[13] L. D. Landau, Sov. Phys. JETP 3 (1956) 920.

[14] V.A. Khodel, V.R. Shaginyan, JETP Lett. 51 (1990) 553.

[15] V.A. Khodel, V.R. Shaginyan, V.V. Khodel, Phys. Rep. 249 (1994) 1.

[16] G. E. Volovik, JETP Lett. 53 (1991) 222.

[17] V.A. Khodel, V.R. Shaginyan, Condens. Matter Theories 12 (1997) 222.

[18] V.A. Khodel, V.R. Shaginyan, JETP Lett. 55 (1992) 110.

[19] V.A. Khodel, V.R. Shaginyan, M.V. Zverev, JETP Lett. 65 (1997) 253.

[20] M.V. Zverev, V.A. Khodel, V.R. Shaginyan, JETP 82 (1996) 567.

[21] V.M. Galitsky, V.A. Khodel, cond-mat/0308203.

[22] V.M.Yakovenko, V.A. Khodel, JETP Lett. 78 (2003) 398; cond-mat/0308380.

[23] V.R. Shaginyan, JETP Lett. 77 (2003) 99; V.R. Shaginyan, JETP Lett. 77 (2003) 178.

[24] A. Casey et al., J. Low Temp. Phys. 113 (1998) 293.

[25] A.A. Shashkin et al., Phys. Rev. B 66 (2002) 073303.

[26] A. Casey et al., Phys. Rev. Lett. 90 (2003) 115301.

[27] J. Boronat et al., Phys. Rev. Lett. 91 (2003) 085302.

[28] Y. Zhang, S. Das Sarma, cond-mat/0312565.

[29] R. Asgari, B. Davoudi, B. Tanatar, Solid St. Comm. in press; cond-mat/0401289.

[30] J.W. Clark, V.A. Khodel, M.V. Zverev, V.M. Yakovenko, Phys. Rep. 391 (2004) 123.

[31] I.E. Dzyaloshinskii, J. Phys. I (France) 6 (1996) 119.

[32] V.Yu. Irkhin, A.A. Katanin, M.I. Katsnelson, Phys. Rev. Lett. 89 (2002) 076401.

[33] D. Lidsky, J. Shiraishi, Y. Hatsugai, M. Kohmoto, Phys. Rev. B 57 (1998) 1340.

[34] E.M. Lifshitz, L.P. Pitaevskii, Statistical Physics, Part 2, Butterworth-Heinemann, 1999.

[35] L. Zhu, M. Garst, A. Rosch, Q. Si, Phys. Rev. Lett. 91 (2003) 066404.

[36] S.L. Bud'ko, E. Morosan, P.C. Canfield, Phys. Rev. 69 (2004) 014415.

[37] D. Takahashi et al., Phys. Rev. B 67 (2003) 180407(R).

[38] Yu.G. Pogorelov, V.R. Shaginyan, JETP Lett. 76 (2002) 532.

[39] A.J. Bennet, M.J. Rice, Phys. Rev. 185 (1969) 968.

[40] J. Paglione, cond-mat/0404269.

[41] S.A. Grigera et al., Science 294 (2001) 329.

[42] C. Capan et al., cond-mat/0404333.

[43] M.Ya. Amusia, V.R. Shaginyan, JETP Lett. 76 (2002) 651. 
[44] M.Ya. Amusia, V.R. Shaginyan, JETP Lett. 77 (2003) 671.

[45] M.Ya. Amusia, V.R. Shaginyan, Phys. Lett. A 315 (2003) 288.

[46] A. Paramekanti, M. Randeria, N. Trivedi, Phys. Rev. Lett. 87 (2001) 217002; A. Paramekanti, M. Randeria, N. Trivedi, cond-mat/0305611.

[47] P.W. Anderson et al., cond-mat/0311467. 